\title{
Aggressive Rosette Forming Glioneuroma: Case Report and Review of the Literature
}

\author{
Michael Frelinghuysen ${ }^{1}$, Francisco Luna ${ }^{2}$, Loreto Spencer ${ }^{3}$, Sebastián Vigueras ${ }^{2}$, Fernando Andrade ${ }^{4}$, Ilson \\ Sepúlveda ${ }^{5} \&$ Tamara Gonzalez ${ }^{1}$ \\ ${ }^{1}$ Department of Radiation Oncology. Hospital Clínico Regional Concepción, Concepción, Chile \\ ${ }^{2}$ Neurosurgery Department, Hospital Clínico Regional Concepción, Concepción, Chile \\ ${ }^{3}$ Department of Pathology Universidad de Concepción, Concepción, Chile \\ ${ }^{4}$ Department of Radiology, Neuroradiology section Hospital Clínico Regional de Concepción, Concepción, \\ Chile \\ ${ }^{5}$ Maxillofacial Radiologist, Otolaryngology and Head and Neck Department, Hospital Clínico Regional de \\ Concepción, Concepción, Chile \\ Correspondence to: Michael Frelinghuysen, Radiation Oncology, Oncology Service, Hospital clinic regional de \\ Concepción, Concepción, Chile. Tel: 56-965-772-994. E-mail: mfrelinghuysen@gmail.com
}

Received: May 18, 2014 Accepted: July 1, 2014 Online Published: July 24, 2014

doi:10.5539/cco.v3n2p10 URL: http://dx.doi.org/10.5539/cco.v3n2p10

\begin{abstract}
We report the case of a patient who presented a pineal tumor. A partial resection was performed. The biopsy showed a glioneuroma rosette forming tumor, considered as WHO grade I. The patient was observed for 13 months and experienced significant tumor growth. Radiotherapy was performed. Instead of scarce evidence of the topic and treatment modalities in this type of tumors; radiotherapy was performed. The patient has been stable and asymptomatic.
\end{abstract}

Keywords: pineal, tumor, partial, resection, radiotherapy

\section{Introducation}

The rosette-forming glioneuronal tumor (RGNT) of the fourth ventricle tumor of the glioneuronal family (Komori, 2002). The clinical presentation, natural history, and treatment response of these tumors has been unclear as there are no significant series of a sizeable population with long-term follow-up (Solis, 2011; Marhold, 2008).

Classically, has been described as WHO I grade neoplasms with indolent biologic behaviour. The optimal management is surgical resection, mainly in symptomatic patients cases where tumor is causing mass effect over neighboring structures (Zhang, 2013). Adjuvant treatments are supported by few data and their role is unknown. We present the case of a rosette forming glioneuroma tumor with aggressive behavior that doubled size after 16 months of follow up after partial resection achieving decompression of neural structures.

\section{Case Report}

The present case refers to a previously healthy 48 years old woman who began with mild headache for 2 weeks. Brain MRI showed an expansive cystic-solid heterogeneous contrast enhancement mass of $2,4 \mathrm{~cm}$ in the pineal region with mass effect over mesencephalon and cuadrigeminal plate. Tumor markers, including beta-human chorionic gonadotropin, and alpha-fetoprotein were not significantly elevated in both the cerebrospinal fluid (CSF). A partial resection of the tumor by endoscopic approach and III cisternoventriculostomy was approached because of thalamic invasion.

The tumor specimen was analyzed by pathology department of Universidad de Concepcion and the case was consulted to neuropathologists of the MD Anderson cancer center. The final biopsy report was Rosette forming glioneuronal tumor, Ki67 negative, GFAP positive. The patient was evaluated then by an interdisciplinary oncology committee (neurosurgery, radiation oncology, pathology and medical oncology) and the management decision based on the evidence reported was observation, mainly due to the indolent behavior reported in the literature. 
Seven months later the patient persisted asymptomatic and a new MRI described the same tumor mass with cystic solid component of $3,3 * 3,1 * 3,3 \mathrm{~cm}$ with mass effect over thalamus, mesencephalon and third ventricle. The case was consulted in a our neuro-oncology committee and also consulted with experienced neuro-oncologists from Medical University of Vienna in Austria, Erasmus MC Brain tumor center in Rotterdam, Netherlands and University of Maryland Medical Center, USA. The final decision was conformal radiotherapy over the tumor mass. She received $54 \mathrm{~Gy}$ in 30 fractions of $1.8 \mathrm{~Gy}$ per fraction prescribed to $100 \%$ isodose line.

Radiotherapy treatment was done using 7 conformal fields to GTV surrounded by $5 \mathrm{~mm}$ PTV. Maximum dose to the brainstem was $54 \mathrm{~Gy}$ in less than $10 \mathrm{cc}$ of the total volume delineated on CT/MRI fusion. The patient evoluted asymptomatic. MRI showed stability of growth and areas of necrosis. The plan is follow up with periodic images in the case the patient remains asymptomatic.
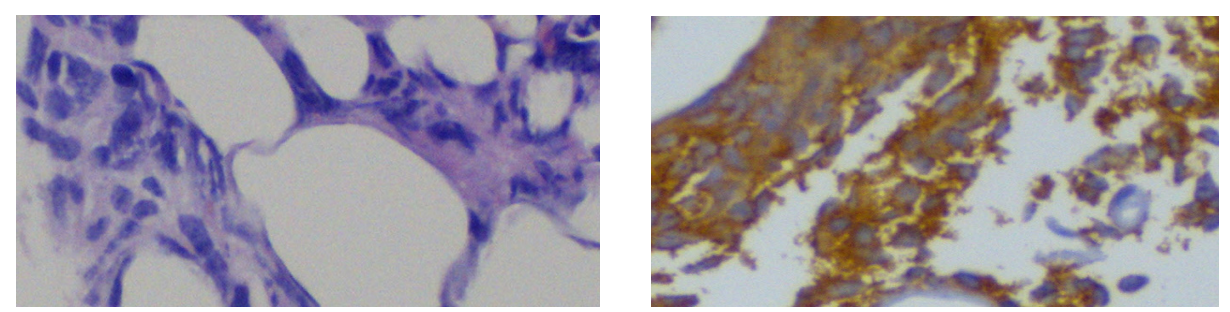

Figure 1. HE tinction showing classic rosettes. The second picture shows positivity to synaptophysin

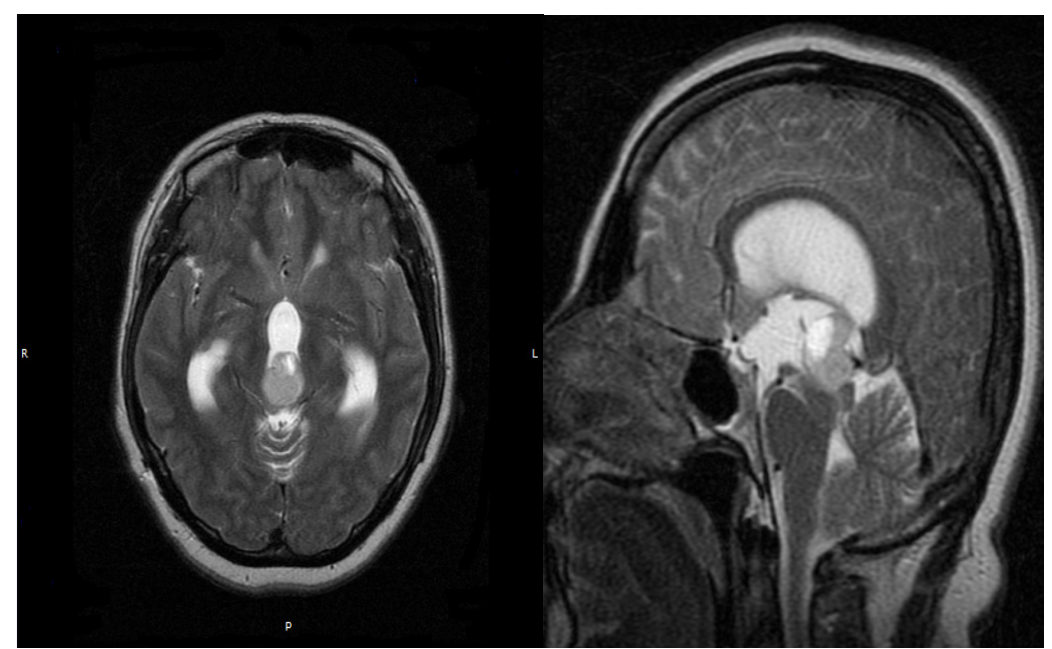

Figure 2. Initial MRI in T2 FSE sequence shows a lesion of approximately $2,4 \mathrm{~cm}$

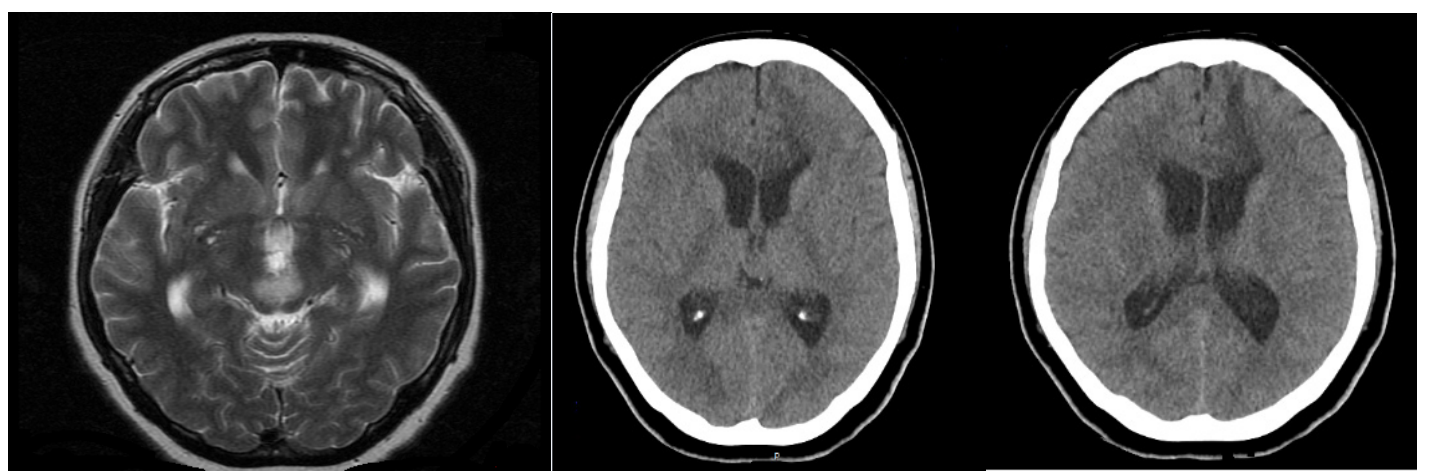

Figure 3. Immediate post-surgery MRI and CT images show the tumor after partial resection 


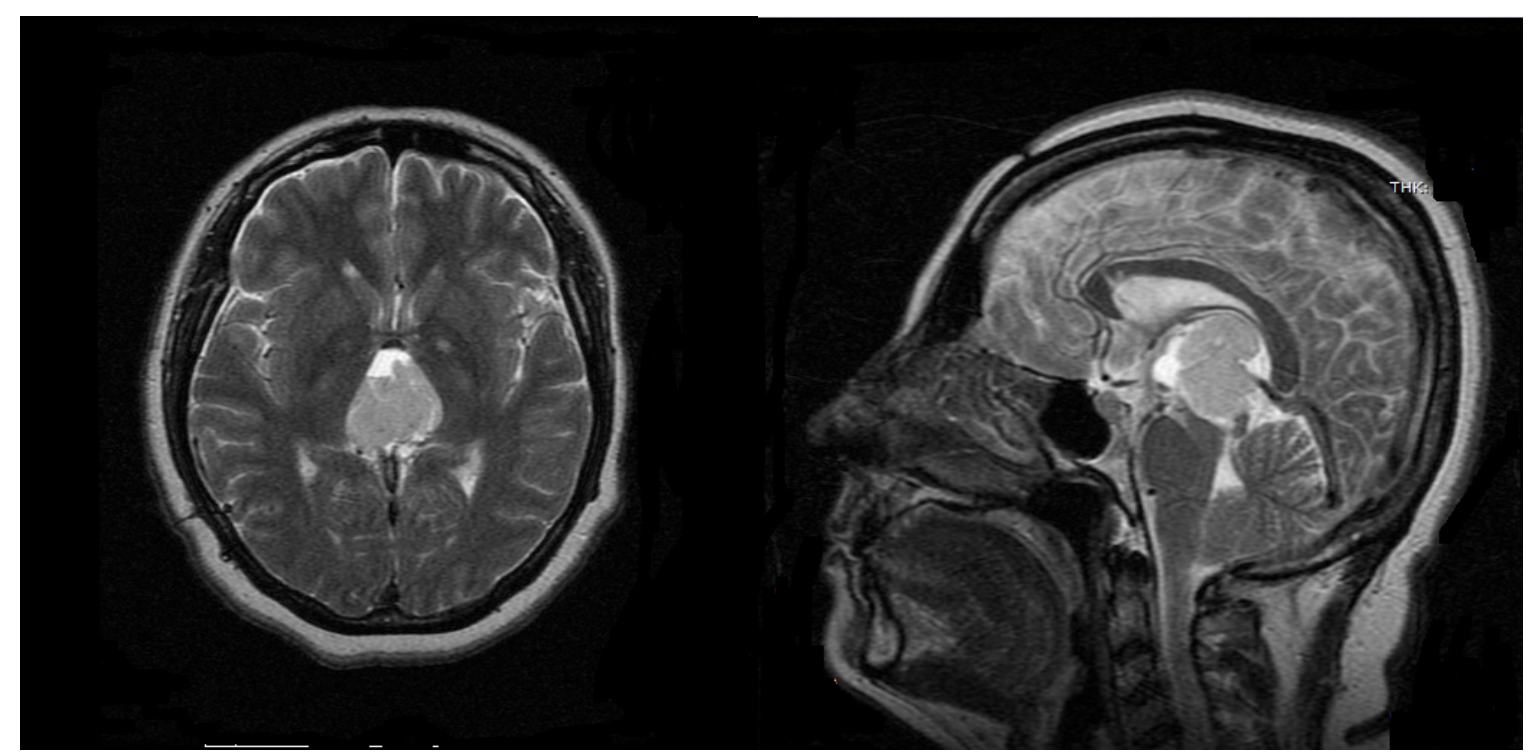

Figure 4. The tumor 13 months after initial diagnosis measuring $36 \mathrm{~mm}$ in major axis. T2 FSE MRI sequences shown denote a clear progression and volume increase

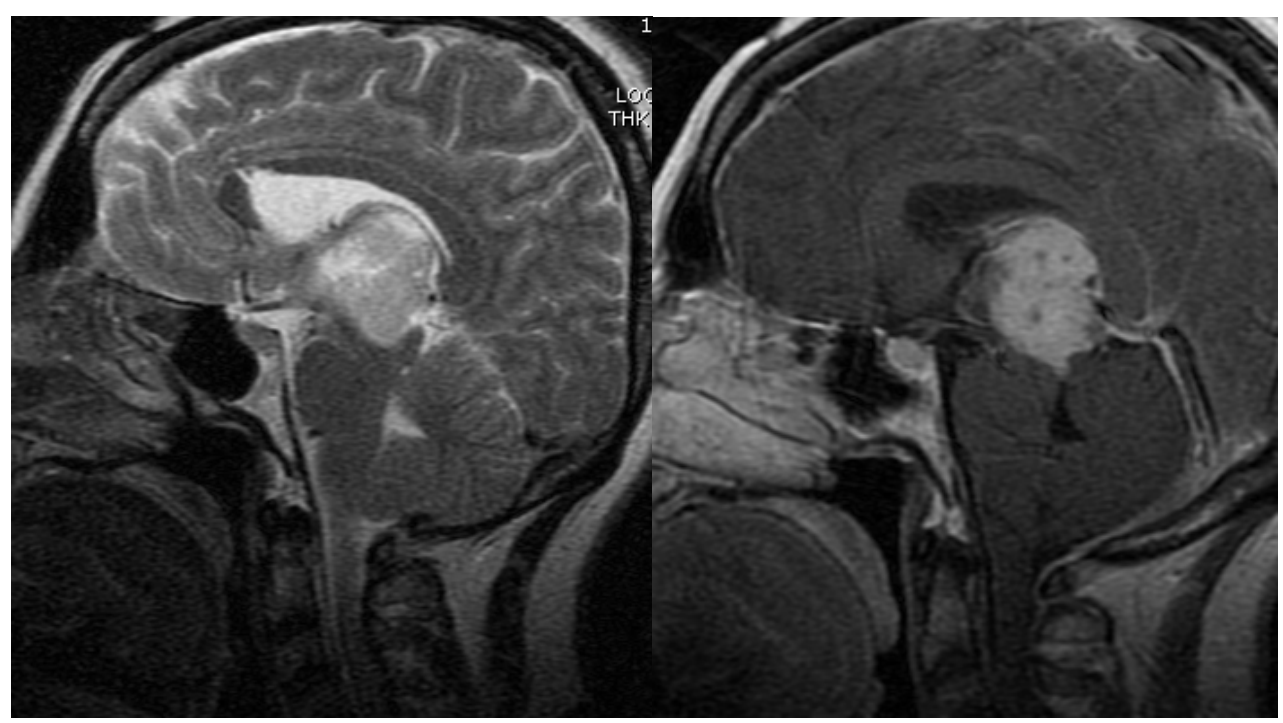

Figure 5. MRI after Radiotherapy treatment shows the stable tumor growth with certain areas of necrosis 

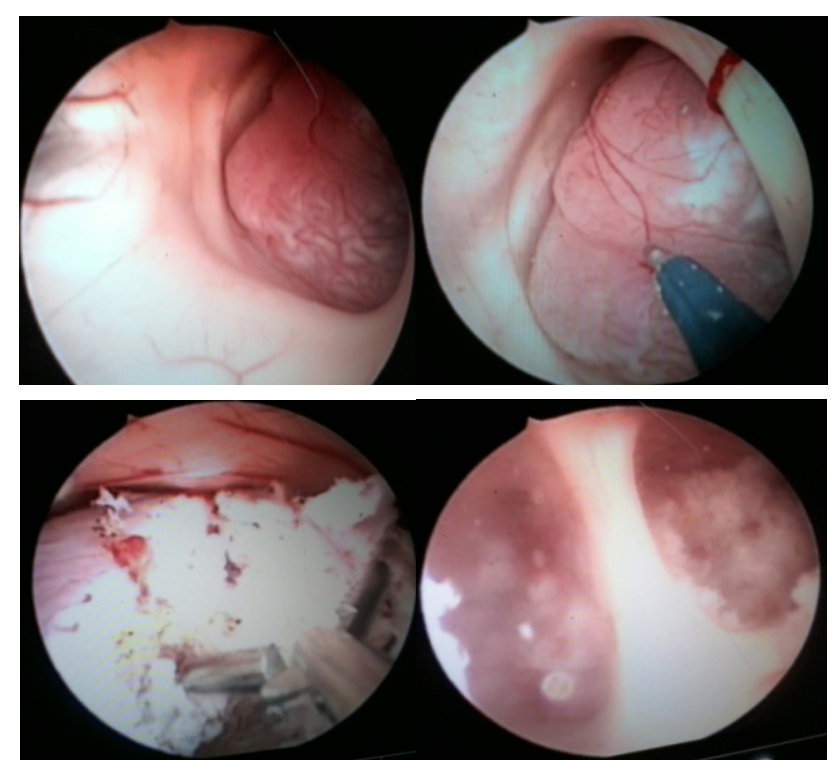

Figure 6. Four phases of endoscopic partial resection
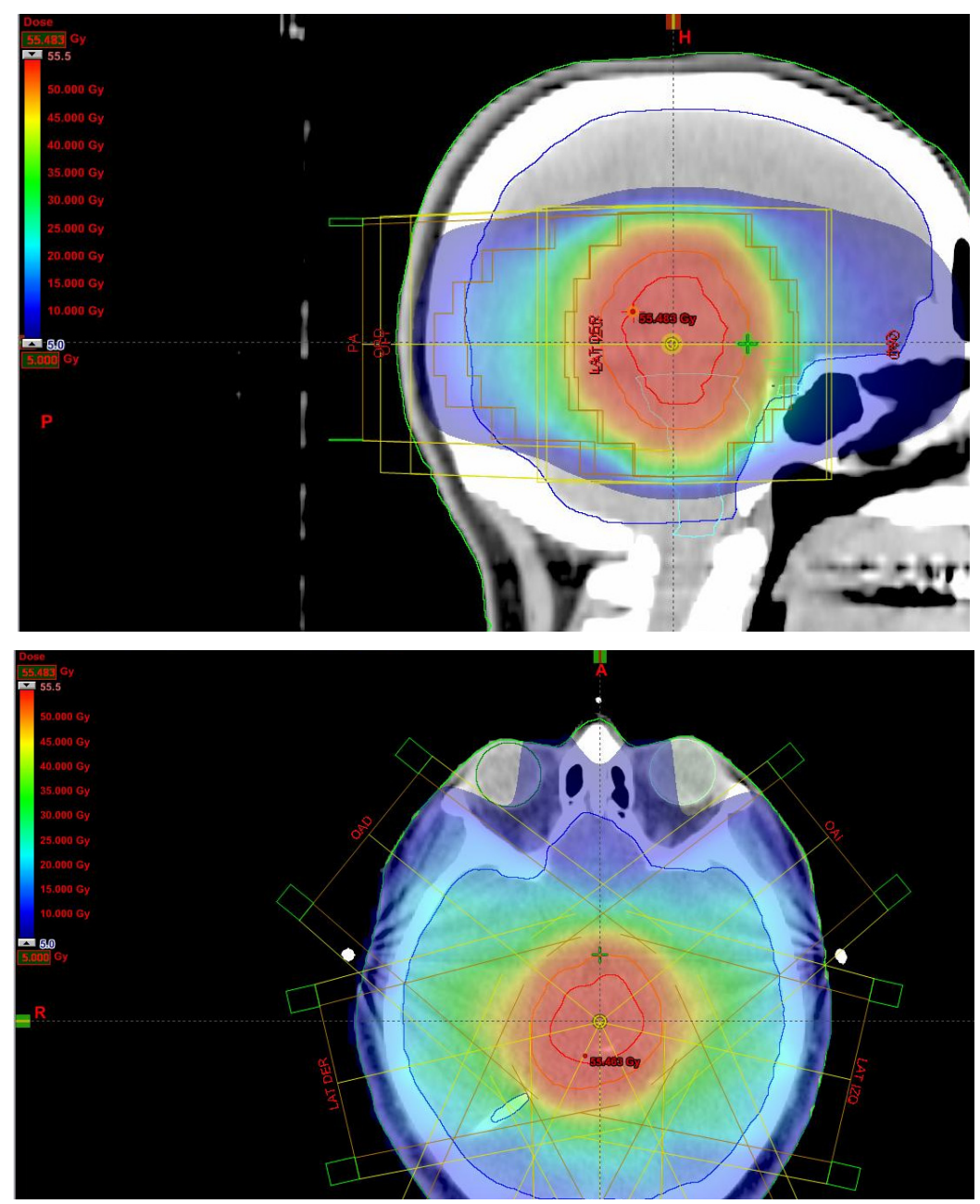

Figure 7. Conformal Radiation Planning. Seven conformational fields prescribed to the isocenter (ICRU point) at $100 \%$ isodose for a total dose of $54 \mathrm{~Gy}$ in 1,8 Gy/fraction showed in an axial and sagittal plane 


\section{Discussion}

Rosette-forming glioneuronal tumor (RGNT) of the fourth ventricle was first described in 2002 (Komori, 2002). Is a rare type of tumor that arises from the walls of the fourth ventricle. It presents more commonly in the third decade and mostly occur in females (Zhang, 2013). Most signs and symptoms associated are typically related to obstructive hydrocephalus and are dependent on the location of the RGNT within the fourth ventricle and the extension to surrounding structures (Marhold, 2008)

Most RGNT arises from the subependymal plate of the fourth ventricle floor and invade dorsally into the cerebellar vermis or ventrally into the brainstem. MRI has been the gold standard to evaluate these tumors and usually presents isointense or hypointense on T1-weighted and hyperintense on T2-weighted images Imaging may also reveal satellite lesions in other parts of the brain and ventricular system (Marhold, 2008).

Many differential diagnosis includes dysembryoplastic neuroepithelial tumor, gliomas, choroid plexus papilloma, , ependymoma, and primitive neuroectodermal tumor (PNET) (Sarrazin, 2006).

RGNT display neurocytic and a glial components and is postulated that derives from pluripotent cells of the subependymal plate (Komori, 2002). The neurocytic component consists of uniform neurocytes that form neurocytic rosettes around vessels, while the glial component resembles low grade gliomas and stains positive for GFAP (Matyja, 2008).

Primary resection is the optimal first-line therapy for these tumors. In our case, the mass contacted the thalamus and mescencephalon, so the complete resection was considered very rough and challenging. Despite gross tumor resection is desirable; the risk must be balanced with functional preservation and sequelae. Most common postoperative complications are ataxia, cranial nerve palsy (CN VI, VII) and diplopia. Cerebellar mutism has also been described in the literature (Tan, 2008; Li, 2009).

Adjuvant treatments as chemotherapy and radiotherapy have not been typically used after resection Radiation has been reported in cases of documented progression by imaging or progressive symptoms (Zhang, 2013; Marhold, 2008; Luan, 2010; Wang, 2009).

We decided to administer to the patient $54 \mathrm{~Gy}$ in $1.8 \mathrm{~Gy} / \mathrm{fx}$ after initial resection due to progressive increasing size of the residual tumor and mild progressive symptoms as nausea and ataxia. The dose was based on the doses reported in the literature (Zhang, 2013; Solis, 2011), however, there is no standard dose. Most reported doses are 54 and 55,8 Gy in $1.8 \mathrm{~Gy} /$ fraction. Radiosurgery and fractionated stereotactic treatments are not reported in this tumors have not been reported to be used in RGNT.

To conclude, RGNT of the fourth ventricle is a novel and rare type of glioneuronal tumor. We presented an aggressive case where we had to use a combined modality treatment. Gross tumor resection in the ideal to accomplish, however, aggressive approach must be balanced against complications and morbidity. Radiotherapy in certain cases might be useful as salvage therapy or progressive disease. Further publications of diagnosis and management of this family of tumors accompanied with research of the role of different and varied biomarkers would better elucidate natural history and prognosis of this disease, as the present case where in spite of being considered WHO I histology, the tumor showed an aggressive behavior, doubling size during 16 months of follow up.

\section{Conflict of Interest}

The authors declare no conflict of interest.

\section{References}

Fushimi, Y., Miyasaki, A., Taki, H., Aoyama, K., Hirato, J., Kanagaki, M., \& Togashi, K. (2011). Rosette-forming glioneuronal tumor of the fourth ventricle with bilateral olivary degeneration. Japanese journal of radiology, 29(6), 445-448. http://dx.doi.org/10.1007/s11604-011-0566-x

Joseph, V., Wells, A., Kuo, Y. H., Halcrow, S., Brophy, B., Scott, G., ... Blumbergs, P. C. (2009). The 'rosette forming glioneuronal tumor' of the fourth ventricle. Neuropathology, 29(3), 309-314. http://dx.doi.org/10.1111/j.1440-1789.2008.00953.x

Komori, T., Scheithauer, B. W., \& Hirose, T. (2002). A rosette-forming glioneuronal tumor of the fourth ventricle: infratentorial form of dysembryoplastic neuroepithelial tumor?. The American journal of surgical pathology, 26(5), 582-591. http://dx.doi.org/10.1097/00000478-200205000-00004

Li, Y. M., Li, W. Q., Pan, Y., Lu, Y. C., Long, N. Y., Tao, X. F., \& Yu, H. Y. (2009). Rosette-forming glioneuronal tumour of the fourth ventricle with previous intratumoural haemorrhage: case report and review of the 
literature. Journal of International Medical Research, 37(3), 958-966. http://dx.doi.org/10.1177 $/ 147323000903700342$

Luan, S., Zhuang, D., Sun, L., \& Huang, F. P. (2010). Rosette-forming glioneuronal tumor (RGNT) of the fourth ventricle: case report and review of literature. Clinical neurology and neurosurgery, 112(4), 362-364. http://dx.doi.org/10.1016/j.clineuro.2010.01.006

Marhold, F., Preusser, M., Dietrich, W., Prayer, D., \& Czech, T. (2008). Clinicoradiological features of rosette-forming glioneuronal tumor (RGNT) of the fourth ventricle: report of four cases and literature review. Journal of neuro-oncology, 90(3), 301-308. http://dx.doi.org/10.1007/s11060-008-9661-y

Matyja, E., Grajkowska, W., Nauman, P., Ozieblo, A., \& Bonicki, W. (2011). Rosette - forming glioneuronal tumor of the fourth ventricle with advanced microvascular proliferation-a case report. Neuropathology, 31(4), 427-432. http://dx.doi.org/10.1111/j.1440-1789.2010.01168.x

Sarrazin, J. L. (2006). Infratentorial tumors. J Radiol, 87(6), 748-763. http://dx.doi.org/10.1016/S02210363(06)74085-9

Solis, O. E., Mehta, R. I., Lai, A., Mehta, R. I., Farchoukh, L. O., Green, R. M., ... Yong, W. H. (2011). Rosette-forming glioneuronal tumor: a pineal region case with IDH1 and IDH2 mutation analyses and literature review of 43 cases. Journal of neuro-oncology, 102(3), 477-484. http://dx.doi.org/10.1007/s11060-010-0335-1

Tan, C. C., Gonzales, M., \& Veitch, A. (2008). Clinical implications of the infratentorial rosette-forming glioneuronal tumor: case report. Neurosurgery, 63(1), E175-E176. http://dx.doi.org/10.1227/ 01.NEU.0000335085.00718.92

Wang, Y., Xiong, J., Chu, S. G., Liu, Y., Cheng, H. X., Wang, Y. F., ... Mao, Y. (2009). Rosette-forming glioneuronal tumor: report of an unusual case with intraventricular dissemination. Acta neuropathologica, 118(6), 813-819. http://dx.doi.org/10.1007/s00401-009-0569-1

Zhang, J., Babu, R., McLendon, R. E., Friedman, A. H., \& Adamson, C. (2013). A comprehensive analysis of 41 patients with rosette-forming glioneuronal tumors of the fourth ventricle. Journal of Clinical Neuroscience, 20(3), 335-341. http://dx.doi.org/10.1016/j.jocn.2012.09.003

\section{Copyrights}

Copyright for this article is retained by the author(s), with first publication rights granted to the journal.

This is an open-access article distributed under the terms and conditions of the Creative Commons Attribution license (http://creativecommons.org/licenses/by/3.0/). 